The Journal of Nonlinear $\mathbf{S}_{\text {cience and }}$ Applications http://www.tjnsa.com

\title{
GENERALIZED CONTRACTIONS AND COMMON FIXED POINT THEOREMS CONCERNING $\tau$-DISTANCE
}

\author{
A. BAGHERI VAKILABAD ${ }^{1}$, S. MANSOUR VAEZPOUR ${ }^{2}$
}

\begin{abstract}
In this paper we consider the generalized distance, present a generalization of Ćirić's generalized contraction fixed point theorems on a complete metric space and investigate a common fixed point theorem about a sequence of mappings concerning generalized distance.
\end{abstract}

\section{INTRODUCTION AND PRELIMINARY}

In order to generalization of Banach's contraction principle, Ćirić introduced generalized contraction([16]). In 2001 Suzuki introduced the concept of $\tau$-distance, a generalization of both $w$-distance ([3]) and Tataru's distance([13]), on a metric space, and discussed it's properties and improved the generalization of Banach's contraction principle , Caristi's fixed point theorem, Downing-Kirk's theorem, Ekeland's variational principal, Hamilton-Jacobi equation, the nonconvex minimization theorem according Takahashi and several fixed point theorems for contractive mapping with respect to $w$-distanc, See $([7],[8],[9],[10],[11,[6],[12,[13])$. In this paper using the $\lambda$-generalized contraction and $\tau$-distance we prove some fixed point theorems. Also, we investigate a sequence of maps which satisfy a common condition of generalized contraction type.

At first we recall some definitions and lemmas which will be used later.

Definition 1.1. ([8]) Let $X$ be a metric space with metric d. A function $p: X \times X \rightarrow[0, \infty)$ is called $\tau$-distance on $X$ if there exist a function $\eta: X \times[0, \infty) \rightarrow[0, \infty)$ such that the following are satisfied:

$\left(\tau_{1}\right) p(x, z) \leq p(x, y)+p(y, z)$ for all $x, y, z \in X$

$\left(\tau_{2}\right) \eta(x, 0)=0$ and $\eta(x, t) \geq t$ for all $x \in X$ and $t \in[0, \infty)$, and $\eta$ is

Date: Revised : 12 Nov. 2009.

2000 Mathematics Subject Classification. 24H25; 54E50; 54H25 .

Key words and phrases. Common fixed point; $\tau$-distance ; generalized contraction. 
concave and continuous in it's second variable;

$\left(\tau_{3}\right) \lim _{n} x_{n}=x$ and $\lim _{n} \sup \left\{\eta\left(z_{n}, p\left(z_{n}, x_{m}\right)\right): m \geq n\right\}=0$, imply $p(w, x) \leq \liminf _{n} p\left(w, x_{n}\right)$ for all $w \in X$;

$\left(\tau_{4}\right) \lim _{n} \sup \left\{p\left(x_{n}, y_{m}\right): m \geq n\right\}=0$ and $\lim _{n} \eta\left(x_{n}, t_{n}\right)=0$, imply $\lim _{n} \eta\left(y_{n}, t_{n}\right)=0$

$\left(\tau_{5}\right) \lim _{n} \eta\left(z_{n}, p\left(z_{n}, x_{n}\right)\right)=0$ and $\lim _{n} \eta\left(z_{n}, p\left(z_{n}, y_{n}\right)\right)=0$, imply $\lim _{n} d\left(x_{n}, y_{n}\right)=0$.

It can be replaced $\left(\tau_{2}\right)$ by the following $\left(\tau_{2}\right)^{\prime}$.

$\left(\tau_{2}\right)^{\prime} \inf \{\eta(x, t): t>0\}=0$ for all $x \in X$, and $\eta$ is nondecreasing in its second variable. The best well-known $\tau$-distances are the metric function $d$ and $w$-distances. If $p$ be a $w$ distance on the metric space $(X, d)$ and a function $\eta$ from $: X \times[0, \infty)$ into $[0, \infty)$ given by $\eta(x, t)=t$, for all $x \in X$, then it is easy to check that $p$ is a $\tau$-distance.

Let $(X, d)$ be a metric space and $p$ be a $\tau$-distance on $X$. A sequence $\left\{x_{n}\right\}$ in $X$ is called $p$-Cauchy if there exists a function $\eta: X \times[0, \infty) \rightarrow[0, \infty)$ satisfying $\left(\tau_{2}\right)-\left(\tau_{5}\right)$ and a sequence $z_{n}$ in $X$ such that $\lim _{n} \sup \left\{\eta\left(z_{n}, p\left(z_{n}, x_{m}\right)\right): m \geq n\right\}=0$.

The following lemmas are essential for next sections.

Lemma 1.2. ([7]) Let $(X, d)$ be a metric space and $p$ be a $\tau$-distance on $X$. If $\left\{x_{n}\right\}$ is a $p$-Cauchy sequence, then it is a Cauchy sequence. Moreover if $\left\{y_{n}\right\}$ is a sequence satisfying $\lim _{n} \sup \left\{p\left(x_{n}, y_{m}\right): m>n\right\}=0$, then $\left\{y_{n}\right\}$ is also $p$-Cauchy sequence and $\lim _{n} d\left(x_{n}, y_{n}\right)=0$.

Lemma 1.3. ([7]) Let $(X, d)$ be a metric space and $p$ be a $\tau$-distance on $X$. If $\left\{x_{n}\right\}$ in $X$ satisfies $\lim _{n} p\left(z, x_{n}\right)=0$ for some $z \in X$, then $x_{n}$ is a $p$-Cauchy sequence. Moreover if $\left\{y_{n}\right\}$ in $X$ also satisfies $\lim _{n} p\left(z, y_{n}\right)=0$, then $\lim _{n} d\left(x_{n}, y_{n}\right)=0$. In particular, for $x, y, z \in X$, $p(z, x)=0$ and $p(z, y)=0$ imply $x=y$.

Lemma 1.4. ([7]) Let $(X, d)$ be a metric space and $p$ be a $\tau$-distance on $X$. If a sequence $\left\{x_{n}\right\}$ in $X$ satisfies $\lim _{n} \sup \left\{p\left(x_{n}, x_{m}\right): m>n\right\}=0$, then $\left\{x_{n}\right\}$ is a $p$-Cauchy sequence. Moreover, if $\left\{y_{n}\right\}$ in $X$ satisfies $\lim _{n} p\left(x_{n}, y_{n}\right)=0$, then $\left\{y_{n}\right\}$ is also $p$-Cauchy sequence and $\lim _{n} d\left(x_{n}, y_{n}\right)=0$.

Remark 1.5. If $p(x, y)=0$ then the equality $x=y$ is not necessarily hold, but $p(x, y)=$ $p(y, x)=0$ imply $x=y$ because $0 \leq p(x, x) \leq p(x, y)+p(y, x)=0$ and hence $p(x, x)=0$. Now by Lemma $1.3 x=y$.

\section{Generalized Contractions}

Throughout this paper we denote by $N$ the set of all positive integer, $R$ real numbers with usual metric and $(X, d)$ be a complete metric space.

Definition 2.1. Let $f$ and $g$ be selfmappings on a complete metric space $X, p$ be a $\tau$-distance on $X$ and $g(X) \subseteq f(X)$. We say $g$ is $\lambda$-generalized contraction (shortly $\lambda$-GC) with respect to $(p, f), \lambda \in(0,1)$, if and only if there exist nonnegative functions $q, r, s, t$, satisfying

$$
\sup _{x, y \in X}\{q(x, y)+r(x, y)+s(x, y)+4 t(x, y)\} \leq \lambda<1
$$


such that for each $x, y \in X$;

$$
\begin{gathered}
\max \{p(f(x), g(y)), p(g(y), f(x))\} \leqq q(x, y) p(x, y)+r(x, y) p(x, f(x)) \\
+s(x, y) p(y, g(y))+t(x, y)[p(x, g(y))+p(y, f(x))] .
\end{gathered}
$$

Example 2.2. a)Let $(X, d)$ be a complete metric space and $p(x, y)=d(x, y)$, then every contraction selfmapping $f$ on $X$ is $\lambda$-GC with respect to $(p, f)$.

b) Let $X=[0,2] \subseteq R$ and

$$
f(x)=g(x)= \begin{cases}\frac{x}{9}, & x \in[0,1] \\ \frac{x}{10}, & x \in(1,2] .\end{cases}
$$

$q(x, y)=\frac{1}{10}, \quad r(x, y)=s(x, y)=\frac{1}{4}, \quad t(x, y)=\frac{1}{11}$ and $p(x, y)=|x-y|$. Then $g$ is $\lambda$-GC with respect to $(p, f)$, but it is not a contraction mapping.

We prove the following lemma which will be used in the next theorem.

Lemma 2.3. Let $x_{0} \in X$. Define the sequence $\left\{x_{n}\right\}$ by

$$
x_{2 n+1}=f\left(x_{2 n}\right), \quad x_{2 n+2}=g\left(x_{2 n+1}\right),
$$

where $f$ and $g$ are selfmappings on $X$ such that $g$ is $\lambda$-GC with respect to $(p, f)$. Then $\left\{x_{n}\right\}$ is a Cauchy sequence.

Proof. Put

and

$$
M_{1}=\max \left\{p\left(x_{2 n+1}, x_{2 n+2}\right), p\left(x_{2 n+2}, x_{2 n+1}\right)\right\}
$$

$$
M_{2}=\max \left\{p\left(x_{2 n}, x_{2 n+1}\right), p\left(x_{2 n+1}, x_{2 n}\right)\right\},
$$

by (2.1), (2.2) and (2.3) we have,

$$
\begin{aligned}
M_{1}= & \max \left\{p\left(f\left(x_{2 n}\right), g\left(x_{2 n+1}\right)\right), p\left(g\left(x_{2 n+1}\right), f\left(x_{2 n}\right)\right)\right\} \\
\leq & \lambda \max \left\{p\left(x_{2 n}, x_{2 n+1}\right), p\left(x_{2 n}, f\left(x_{2 n}\right)\right),\right. \\
& p\left(x_{2 n+1}, g\left(x_{2 n+1}\right)\right), \frac{1}{4}\left[p\left(x_{2 n}, g\left(x_{2 n+1}\right)\right)+p\left(x_{2 n+1}, f\left(x_{2 n}\right)\right]\right\} \\
= & \lambda \max \left\{p\left(x_{2 n}, x_{2 n+1}\right), p\left(x_{2 n}, x_{2 n+1}\right),\right. \\
& \left.p\left(x_{2 n+1}, x_{2 n+2}\right), \frac{1}{4}\left[p\left(x_{2 n}, x_{2 n+2}\right)+p\left(x_{2 n+1}, x_{2 n+1}\right)\right]\right\} \\
= & \lambda M\left(x_{2 n}, x_{2 n+1}\right)
\end{aligned}
$$

where

$$
M\left(x_{2 n}, x_{2 n+1}\right)=\max \left\{p\left(x_{2 n}, x_{2 n+1}\right), p\left(x_{2 n+1}, x_{2 n+2}\right), \frac{1}{4}\left[p\left(x_{2 n}, x_{2 n+2}\right)+p\left(x_{2 n+1}, x_{2 n+1}\right)\right]\right\} .
$$

Now if $M\left(x_{2 n}, x_{2 n+1}\right)=p\left(x_{2 n+1}, x_{2 n+2}\right)$, then we have,

$$
p\left(x_{2 n+1}, x_{2 n+2}\right) \leq \lambda p\left(x_{2 n+1}, x_{2 n+2}\right),
$$

which implies $p\left(x_{2 n+1}, x_{2 n+2}\right)=0$.

If $M\left(x_{2 n}, x_{2 n+1}\right)=\frac{1}{4}\left[p\left(x_{2 n}, x_{2 n+2}\right)+p\left(x_{2 n+1}, x_{2 n+1}\right)\right]$ then,

$$
p\left(x_{2 n+1}, x_{2 n+2}\right) \leq \frac{\lambda}{4}\left[p\left(x_{2 n}, x_{2 n+2}\right)+p\left(x_{2 n+1}, x_{2 n+1}\right)\right],
$$


SO

$$
p\left(x_{2 n+1}, x_{2 n+2}\right) \leq \frac{\lambda}{2} p\left(x_{2 n}, x_{2 n+2}\right) \quad \text { or } \quad p\left(x_{2 n+1}, x_{2 n+2}\right) \leq \frac{\lambda}{2} p\left(x_{2 n+1}, x_{2 n+1}\right) .
$$

If $p\left(x_{2 n+1}, x_{2 n+2}\right) \leq \frac{\lambda}{2} p\left(x_{2 n}, x_{2 n+2}\right)$ since,

$$
\begin{aligned}
\frac{\lambda}{2} p\left(x_{2 n}, x_{2 n+2}\right) & \leq \frac{\lambda}{2}\left[p\left(x_{2 n}, x_{2 n+1}\right)+p\left(x_{2 n+1}, x_{2 n+2}\right)\right] \\
& \leq \frac{\lambda}{2} p\left(x_{2 n}, x_{2 n+1}\right)+\frac{1}{2} p\left(x_{2 n+1}, x_{2 n+2}\right)
\end{aligned}
$$

we have

$$
p\left(x_{2 n+1}, x_{2 n+2}\right) \leq \lambda p\left(x_{2 n}, x_{2 n+1}\right) .
$$

If $p\left(x_{2 n+1}, x_{2 n+2}\right) \leq \frac{\lambda}{2} p\left(x_{2 n+1}, x_{2 n+1}\right)$ since,

$$
\frac{\lambda}{2} p\left(x_{2 n+1}, x_{2 n+1}\right) \leq \frac{\lambda}{2}\left[p\left(x_{2 n+1}, x_{2 n}\right)+p\left(x_{2 n}, x_{2 n+1}\right)\right]
$$

we have

$$
p\left(x_{2 n+1}, x_{2 n+2}\right) \leq \lambda p\left(x_{2 n+1}, x_{2 n}\right) \quad \text { or } \quad p\left(x_{2 n+1}, x_{2 n+2}\right) \leq \lambda p\left(x_{2 n}, x_{2 n+1}\right) .
$$

Therefore in any cases we have;

$$
M_{1} \leq \lambda p\left(x_{2 n+1}, x_{2 n}\right) \text { or } \quad M_{1} \leq \lambda p\left(x_{2 n}, x_{2 n+1}\right) .
$$

Similarly

$$
M_{2} \leq \lambda p\left(x_{2 n-1}, x_{2 n}\right) \text { or } \quad M_{2} \leq \lambda p\left(x_{2 n}, x_{2 n-1}\right)
$$

Continuing this process we have,

$$
p\left(x_{n}, x_{n+1}\right) \leq \lambda \max \left\{p\left(x_{n-1}, x_{n}\right), p\left(x_{n}, x_{n-1}\right)\right\} \leq \ldots \leq \lambda^{n} \max \left\{p\left(x_{0}, x_{1}\right), p\left(x_{1}, x_{0}\right)\right\}
$$

Putting $r\left(x_{0}\right)=\max \left\{p\left(x_{0}, x_{1}\right), p\left(x_{1}, x_{0}\right)\right\}$, then for any $m>n$;

$$
p\left(x_{m}, x_{n}\right) \leq \sum_{k=0}^{m-n-1} p\left(x_{n+k+1}, x_{n+k}\right) \leq \sum_{k=0}^{m-n-1} \lambda^{(n+k)} r\left(x_{0}\right) \leq \lambda^{n} r\left(x_{0}\right)(1-\lambda)^{-1} .
$$

So $\lim \sup _{n}\left\{p\left(x_{m}, x_{n}\right): m \geq n\right\}=0$. Hence by Lemmas 1.2 and $1.4\left\{x_{n}\right\}$ is a Cauchy sequence.

Theorem 2.4. Let $(X, d)$ be a metric space, $p$ be a $\tau$-distance on $X$ and $x_{0} \in X$ and $f$ and $g$ be selfmappings on $X$ such that $g$ is $\lambda$-GC with respect to $(p, f)$. Moreover assume that the following holds:

If $\limsup _{n}\left\{p\left(x_{n}, x_{m}\right): m>n\right\}=0$ and $\lim _{n} p\left(x_{n}, y\right)=0$ then, $\lim _{n} p\left(x_{n}, f\left(x_{n}\right)\right)=0$ implies $f(y)=y$ and $\lim _{n} p\left(x_{n}, g\left(x_{n}\right)\right)=0$ implies $g(y)=y$. Then $f$ and $g$ have a unique common fixed point, namely $z$, such that $p(z, z)=0$ and $(f g)^{n}\left(x_{0}\right) \rightarrow z$ and $(g f)^{n}\left(x_{0}\right) \rightarrow z$. 
Proof. Let $x_{0} \in X$. Define the sequence $\left\{x_{n}\right\}$ by $x_{2 n+1}=f\left(x_{2 n}\right)$ and $x_{2 n+2}=g\left(x_{2 n+1}\right)$. Then by Lemma $2.3\left\{x_{n}\right\}$ is a Cauchy sequence and converges to some point $z \in X$. We show that $f(z)=z$, and $g(z)=z$.

By $\left(\tau_{3}\right)$ we have;

$$
\begin{aligned}
\limsup _{n}\left(p\left(x_{2 n}, f\left(x_{2 n}\right)\right)+p\left(x_{2 n}, z\right)\right) & \leq \limsup _{n}\left(p\left(x_{2 n}, x_{2 n+1}\right)+\liminf _{m \rightarrow \infty} p\left(x_{2 n}, x_{m}\right)\right. \\
& \leq 2 \limsup _{m \geq 2 n} p\left(x_{2 n}, x_{m}\right)=0 .
\end{aligned}
$$

Similarly $\lim \sup _{n}\left(p\left(x_{2 n+1}, g\left(x_{2 n+1}\right)\right)+p\left(x_{2 n+1}, z\right)\right)=0$. Therefore

$$
\limsup _{n}\left\{p\left(x_{n}, x_{m}\right): m>n\right\}=0 \quad \text { and } \quad \lim _{n}\left(x_{n}, z\right)=0 .
$$

So we have,

$$
\lim _{n}\left(p\left(x_{2 n}, f\left(x_{2 n}\right)\right)=0\right.
$$

and

$$
\lim _{n} p\left(x_{2 n}, z\right)=0
$$

Putting $x_{n}^{\prime}=x_{2 n}$, the hypothesis implies $f(z)=z$. With a similar computations we have $g(z)=z$.

Now if we put $x=y=z$ in $(2.2)$ we get $p(z, z) \leq \lambda p(z, z)$ which implies $p(z, z)=0$.

If $u$ be another common fixed point for $f$ and $g$ by using (2.2) we have

$$
\begin{aligned}
\max \{p(z, u), p(u, z)\} & \leqq q(z, u) p(z, u)+r(z, u) p(z, z)+s(z, u) p(u, u) \\
& +t(z, u)[p(z, u)+p(u, z)] \\
& \leq \lambda \cdot \max \left\{p(z, u), p(z, z), p(u, u), \frac{1}{4}[p(z, u)+p(u, z)]\right\} \\
& =\lambda \cdot \max \left\{p(z, u), \frac{1}{4}[p(z, u)+p(u, z)\} .\right.
\end{aligned}
$$

The last equality holds because $p(z, z)=p(u, u)=0$. In any cases this inequalities show that $p(z, u)=p(u, z)=0$ and by Remark $1.5 z=u$.

Note that if $f$ is continuous then, $\left\{x_{n}\right\}$ and $\left\{f\left(x_{n}\right)\right\}$ converge to $y$, implies $f(y)=y$. If $\lim \sup _{n}\left\{p\left(x_{n}, x_{m}\right): m>n\right\}=0, \quad \lim _{n} p\left(x_{n}, y\right)=0$, and $\lim _{n} p\left(x_{n}, f\left(x_{n}\right)\right)=0$, then by Lemma 1.4 we have $\lim _{n} x_{n}=\lim _{n} f\left(x_{n}\right)=y$, but in general it doesn't imply $f(y)=y$. For example, let $X=R$, (real numbers with usual metric), $x_{n}=\frac{n-1}{n}, p=d, y=1$ and $f: R \rightarrow R$ defined by

$$
f(t)= \begin{cases}t, & t \neq 1 \\ 2, & t=1 .\end{cases}
$$

It is possible that $g^{k}$ be $\lambda$-GC with respect to $(p, f)$, for some $k \in N$ and $k>1$, but $g$ is not so.

Example 2.5. Let $X=\{a, b, c\}$ where $a, b, c \in R$ are three distinct real numbers; $f(x)=a$, constant map on $X$, and $g: X \rightarrow X$ is given by $g(a)=a, g(b)=c, g(c)=a$. Put $p=d$. We have $g^{2}=f$, and so $g^{2}$ is $\lambda$-GC with respect to $(p, f)$, but since $g(X) \nsubseteq f(X)$ so $g$ is not $\lambda$-GC with respect to $(p, f)$. 
Corollary 2.6. Let $(X, d)$ be a metric space, $p$ be a $\tau$-distance on $X$ and $x_{0} \in X$ and $f$ and $g$ be selfmappings on $X$ such that $g^{k}$ is $\lambda$-GC with respect to $(p, f)$, for some $k \in N$. Moreover assume that the following holds:

If $\limsup _{n}\left\{p\left(x_{n}, x_{m}\right): m>n\right\}=0$ and $\lim _{n} p\left(x_{n}, y\right)=0$ then, $\lim _{n} p\left(x_{n}, f\left(x_{n}\right)\right)=0$ implies $f(y)=y$ and $\lim _{n} p\left(x_{n}, g^{k}\left(x_{n}\right)\right)=0$ implies $g^{k}(y)=y$. Then $f$ and $g$ have a unique common fixed point.

Proof. By Theorem $2.4 f$ and $g^{k}$ have common fixed point, $z$. Now we have $g^{k}(g(z))=$ $g\left(g^{k}(z)\right)=g(z)$. It follows that $g(z)=z=f(z)$, by uniqueness.

Corollary 2.7. Let $(X, d)$ be a metric space, $p$ be a $\tau$-distance on $X, x_{0} \in X$ and $f$ and $g$ be selfmappings on $X$ such that $g$ is $\lambda$-GC with respect to $(p, f)$. Moreover assume that if $\left\{x_{n}\right\},\left\{f\left(x_{n}\right)\right\}$ and $\left\{g\left(x_{n}\right)\right\}$ converges to $y$, it implies $f(y)=y$ and $g(y)=y$. Then $f$ and $g$ have a unique common fixed point, namely $z$, such that $p(z, z)=0$ and $(f g)^{n}\left(x_{0}\right) \rightarrow z$ and $(g f)^{n}\left(x_{0}\right) \rightarrow z$.

Corollary 2.8. Let $(X, d)$ be a metric space, $p$ be a $\tau$-distance on $X$ and $x_{0} \in X$. Suppose $f$ and $g$ are continuous selfmappings on $X$, and $g$ is $\lambda$-GC with respect to $(p, f)$. Then $f$ and $g$ have a unique common fixed point, namely $z$, such that $p(z, z)=0$ and $(f g)^{n}\left(x_{0}\right) \rightarrow z$ and $(g f)^{n}\left(x_{0}\right) \rightarrow z$.

\section{Sequence of Generalized Contraction Maps}

Throughout this section we prove a common fixed point theorem for a sequence of maps which satisfy a common condition of generalized contraction type. We begin with a lemma.

Lemma 3.1. Let $(X, d)$ be a metric space, $p$ be a $\tau$-distance on $X$. Let $f$ and $f_{0}$ be selfmappings on $X$ such that the following holds:

$$
\begin{gathered}
\max \left\{p\left(f_{0}(x), f(y)\right), p\left(f(y), f_{0}(x)\right)\right\} \leq \lambda \max \left\{p(x, y), p\left(x, f_{0}(x)\right),\right. \\
\left.p(y, f(y)), p(x, f(y)), p\left(y, f_{0}(x)\right)\right\}
\end{gathered}
$$

for some $\lambda \in(0,1)$ and all $x, y \in X$. If $f_{0}(z)=z$ and $p(z, z)=0$, for some $z \in X$, then $f(z)=z$ and $z$ is unique.

Proof. Since $f_{0}(z)=z$, by (3.1) we have

$$
\begin{aligned}
\max \{p(z, f(z)), p(f(z), z)\} & =\max \left\{p\left(f_{0}(z), f(z)\right), p\left(f(z), f_{0}(z)\right)\right\} \\
& \leqq \lambda \max \{p(z, z), p(z, f(z))\}=\lambda p(z, f(z))
\end{aligned}
$$

which implies $p(z, f(z))=0$ and hence by Lemma $1.3 z=f(z)$.

If $v \in X$ be such that $f_{0}(v)=v$ and $p(v, v)=0$ then we have $f(v)=v$ and

$$
\begin{aligned}
p(z, v)=p\left(f_{0}(z), f(v)\right) & \leq \lambda \max \{p(z, v), p(z, z), p(v, v), p(v, z)\} \\
& =\lambda \max \{p(z, v), p(v, z)\} .
\end{aligned}
$$

With similar computation

$$
p(v, z) \leq \lambda \max \{p(z, v), p(v, z)\}
$$

so $p(z, v)=p(v, z)=0$ and by Remark (1.5) $v=z$. 
Theorem 3.2. Let $(X, d)$ be a complete metric space, $p$ be a $\tau$-distance on $X$ and $\left\{f_{n}\right\}$ be a sequence of selfmappings on $X$, such that $f_{0}$ is continuous and for each $x, y \in X$;

$$
\begin{gathered}
\max \left\{p\left(f_{0}(x), f_{n}(y)\right), p\left(f_{n}(y), f_{0}(x)\right)\right\} \leqq \lambda \cdot \max \left\{p(x, y), p\left(x, f_{0}(x)\right),\right. \\
p\left(y, f_{n}(y)\right), \frac{1}{4}\left[p\left(x, f_{n}(y)\right)+p\left(y, f_{0}(x)\right)\right\},
\end{gathered}
$$

whereas $\lambda \in(0,1)$ and $n=0,1,2,3, \ldots$. Then $\left\{f_{n}\right\}$ have a unique common fixed point, namely $z$, such that $p(z, z)=0$.

Proof. Let $x_{0} \in X$. Define the sequence $\left\{x_{n}\right\}$ by

$$
x_{1}=f_{0}\left(x_{0}\right), x_{2}=f_{0}\left(x_{1}\right)=f_{0}^{2}\left(x_{0}\right), \ldots, x_{n}=f_{0}^{n}\left(x_{0}\right), \ldots
$$

We show that $\left\{x_{n}\right\}$ is a Cauchy sequence. By (3.2) we have

$$
\begin{aligned}
& \max \left\{p\left(x_{n}, x_{n-1}\right), p\left(x_{n-1}, x_{n}\right)\right\}=\max \left\{p\left(f_{0}\left(x_{n-1}\right), f_{0}\left(x_{n-2}\right)\right), p\left(f_{0}\left(x_{n-2}\right), f_{0}\left(x_{n-1}\right)\right)\right\} \\
& \quad \leq \lambda \max \left\{p\left(x_{n-2}, x_{n-1}\right), p\left(x_{n-1}, x_{n}\right), \frac{1}{4} p\left(x_{n-2}, x_{n}\right)+p\left(x_{n-1}, x_{n-1}\right)\right\} .
\end{aligned}
$$

We will prove that

$$
\max \left\{p\left(x_{n}, x_{n-1}\right), p\left(x_{n-1}, x_{n}\right)\right\} \leq \lambda \max \left\{p\left(x_{n-1}, x_{n-2}\right), p\left(x_{n-2}, x_{n-1}\right)\right\} .
$$

To show this set $M=\max \left\{p\left(x_{n-2}, x_{n-1}\right), p\left(x_{n-1}, x_{n}\right), \frac{1}{4} p\left(x_{n-2}, x_{n}\right)+p\left(x_{n-1}, x_{n-1}\right)\right\}$.

If $M=p\left(x_{n-1}, x_{n}\right)$ then $p\left(x_{n-1}, x_{n}\right)=0$ and (3.4) holds.

If $M=p\left(x_{n-2}, x_{n-1}\right)$ then $\max \left\{p\left(x_{n}, x_{n-1}\right), p\left(x_{n-1}, x_{n}\right)\right\} \leq \lambda p\left(x_{n-2}, x_{n-1}\right)$ and (3.4) holds

If $\left.M=\frac{1}{4} p\left(x_{n-2}, x_{n}\right)+p\left(x_{n-1}, x_{n-1}\right)\right\}$ then

$4 \max \left\{p\left(x_{n}, x_{n-1}\right), p\left(x_{n-1}, x_{n}\right)\right\} \leq \lambda p\left(x_{n-2}, x_{n}\right)+p\left(x_{n-1}, x_{n-1}\right)$ hence

$$
\begin{aligned}
2 \max \left\{p\left(x_{n}, x_{n-1}\right), p\left(x_{n-1}, x_{n}\right)\right\} & \leq \lambda p\left(x_{n-2}, x_{n}\right) \\
& \leq \lambda p\left(x_{n-2}, x_{n-1}\right)+p\left(x_{n-1}, x_{n}\right)
\end{aligned}
$$

or

$$
\begin{aligned}
2 \max \left\{p\left(x_{n}, x_{n-1}\right), p\left(x_{n-1}, x_{n}\right)\right\} & \leq \lambda p\left(x_{n-1}, x_{n-1}\right) \\
& \leq \lambda p\left(x_{n-1}, x_{n-2}\right)+p\left(x_{n-2}, x_{n-1}\right)
\end{aligned}
$$

which implies

$$
\max \left\{p\left(x_{n}, x_{n-1}\right), p\left(x_{n-1}, x_{n}\right)\right\} \leq \lambda \max \left\{p\left(x_{n-1}, x_{n-2}\right), p\left(x_{n-2}, x_{n-1}\right)\right\},
$$

so in any cases (3.4) holds.

Continuing this process one has,

$$
p\left(x_{n-1}, x_{n}\right) \leq \lambda \max \left\{p\left(x_{n-2}, x_{n-1}\right), p\left(x_{n-1}, x_{n-2}\right)\right\} \leq \ldots \leq \lambda^{n} \max \left\{p\left(x_{0}, x_{1}\right), p\left(x_{1}, x_{0}\right)\right\}
$$

Putting $r\left(x_{0}\right)=\max \left\{p\left(x_{0}, x_{1}\right), p\left(x_{1}, x_{0}\right)\right\}$, for any $m>n$;

$$
p\left(x_{n}, x_{m}\right) \leq \sum_{k=0}^{m-n-1} p\left(x_{n+k}, x_{n+k+1}\right) \leq \sum_{k=0}^{m-n-1} \lambda^{(n+k)} r\left(x_{0}\right) \leq \lambda^{n} r\left(x_{0}\right)(1-\lambda)^{-1} .
$$

So $\lim \sup _{n}\left\{p\left(x_{n}, x_{m}\right): m>n\right\}=0$. Then by Lemma $1.4\left\{x_{n}\right\}$ is a Cauchy sequence, since $X$ is complete metric space there exist some point $z \in X$ such that $\lim _{n} x_{n}=z$. On the other hand continuity of $f_{0}$ implies

$$
f_{0}(z)=f_{0}\left(\lim _{n} x_{n}\right)=\lim _{n}\left(f_{0}\left(x_{n}\right)\right)=\lim _{n}\left(x_{n+1}\right)=z
$$


therefore $f_{0}(z)=z$. By $(3.2)$ we have

$$
p(z, z)=p\left(f_{0}(z), z\right)=p\left(z, f_{0}(z)\right)=p\left(f_{0}(z), f_{0}(z)\right) \leqq \lambda p(z, z),
$$

so $p(z, z)=0$. Then by Lemma $3.1 z$ is a unique fixed point of $f_{0}$ and $f_{n}(z)=z$ for all $n=1,2,3, \ldots$

Note that if the condition of continuity of $f_{0}$ is replaced by the lower semicontinity of $p$ in its first variable, the theorem will be holds too. Because if $p$ be lower semicontinuous in its first variable by (3.2) and triangle inequality we have

$$
\begin{aligned}
p\left(z, f_{0}(z)\right) \leq & p\left(z, x_{n}\right)+p\left(f_{0}\left(x_{n-1}\right), f_{0}(z)\right) \\
\leq & p\left(z, x_{n}\right)+\lambda \max \left\{p\left(z, x_{n-1}\right), p\left(z, f_{0}(z)\right), p\left(x_{n-1}, f_{n}\left(x_{n-1}\right)\right),\right. \\
& \frac{1}{4}\left[p\left(z, f_{n}\left(x_{n-1}\right)\right)+p\left(x_{n-1}, f_{0}(z)\right)\right\} \\
\leq & p\left(z, x_{n}\right)+\lambda \cdot \max \left\{p\left(z, x_{n-1}\right), p\left(z, f_{0}(z)\right), p\left(x_{n-1}, x_{n}\right),\right. \\
& \frac{1}{4}\left[p\left(z, x_{n}\right)+p\left(x_{n-1}, f_{0}(z)\right)\right] \\
\leq & p\left(z, x_{n}\right)+\lambda \cdot\left[p\left(z, x_{n-1}\right)+p\left(z, f_{0}(z)\right)+p\left(x_{n-1}, x_{n}\right)+p\left(x_{n}, z\right)\right]
\end{aligned}
$$

hence

$$
p\left(z, f_{0}(z)\right) \leq \frac{1}{1-\lambda}\left[p\left(z, x_{n}\right)+\lambda\left[p\left(z, x_{n-1}\right)+p\left(x_{n-1}, x_{n}\right)+p\left(x_{n}, z\right)\right]\right]
$$

By $\left(\tau_{3}\right)$

$$
\left(p\left(x_{n}, z\right)\right) \leq \liminf _{m}\left(p\left(x_{n}, x_{m}\right) \leq \lambda^{n} r\left(x_{0}\right)(1-\lambda)^{-1}\right.
$$

so $\lim _{n}\left(p\left(x_{n}, z\right)\right)=0$, moreover by construction $\lim _{n}\left(p\left(x_{n-1}, x_{n}\right)\right)=0$. Since $p$ is lower semicontinuous in its first variable we have

$$
\lim _{n} p\left(z, x_{n}\right)=\lim _{n} p\left(z, x_{n-1}\right)=0,
$$

therefore $p\left(z, f_{0}(z)\right)=0$. On the other hand

$$
\begin{aligned}
p\left(f_{0}(z), z\right) \leq & p\left(x_{n}, z\right)+p\left(f_{0}(z), f_{0}\left(x_{n-1}\right)\right) \\
\leq & p\left(x_{n}, z\right)+\lambda \max \left\{p\left(z, x_{n-1}\right), p\left(x_{n-1}, f_{0}\left(x_{n-1}\right)\right), p\left(z, f_{0}(z)\right),\right. \\
& \left.\frac{1}{4}\left[p\left(x_{n-1}, f_{0}(z)\right)+p\left(z, f_{0}\left(x_{n-1}\right)\right)\right]\right\} \\
\leq & p\left(x_{n}, z\right)+\lambda \max \left\{p\left(z, x_{n-1}\right), p\left(x_{n-1}, x_{n}\right), p\left(z, f_{0}(z)\right),\right. \\
& \left.\frac{1}{4}\left[p\left(x_{n-1}, f_{0}(z)\right)+p\left(z, x_{n}\right)\right]\right\} \\
\leq & p\left(x_{n}, z\right)+\lambda\left[p\left(z, x_{n-1}\right)+p\left(x_{n-1}, x_{n}\right)+p\left(x_{n}, z\right)+p\left(z, f_{0}(z)\right)\right] .
\end{aligned}
$$

Hence $p\left(f_{0}(z), z\right)=0$ and so $f_{0}(z)=z$ and we have the following theorem:

Theorem 3.3. Let $(X, d)$ be a complete metric space, $p$ be a $\tau$-distance on $X$ such that $p$ is lower semicontinuous in its first variable and $\left\{f_{n}\right\}$ be a sequence of selfmappings on $X$, satisfying

$$
\max \left\{p\left(f_{0}(x), f_{n}(y)\right), p\left(f_{n}(y), f_{0}(x)\right)\right\} \leqq \lambda \cdot \max \left\{p(x, y), p\left(x, f_{0}(x)\right)\right.
$$




$$
p\left(y, f_{n}(y)\right), \frac{1}{4}\left[p\left(x, f_{n}(y)\right)+p\left(y, f_{0}(x)\right)\right\} .
$$

for each $x, y \in X, \lambda \in(0,1)$ and $n=0,1,2,3, \ldots$. Then $\left\{f_{n}\right\}$ have a unique common fixed point, namely $z$, such that $p(z, z)=0$.

\section{REFERENCES}

[1] M. Alimohammadi and M. Ramzanzadeh, On $\Phi$-Fixed point for maps on uniform spaces, J. of Nonlinear Science and Applications, 1(4)(2008), 241-243.

[2] A. Azam and M. Arshad, Kannan fixed point theorem on generalized metric spaces, J. of Nonlinear Science and Applications, 1(1)(2008), 45-48.

[3] O. Kada, T. Suzuki and W. Takahashi, Nonconvex Minimization theorems and fixed point theorems in complete metric spaces, Math. Japon, 44 (1996), 381-391.

[4] D. Mihet, On Kannan fixed point principle in generalized metric spaces, J. of Nonlinear Science and Applications, 2(2)(2009), 92-96.

[5] I. R. Sarma, J. M. Rao2, and S. S. Rao, Contractions over generalized metric spaces, J. of Nonlinear Science and Applications, 2(3)(2009), 180-182.

[6] N. Shioji, T. Suzuki and W. Takahashi, Contractive mappings, Kannan mappings and metric completness, Proc. Amer. Math. Soc, 126 (1998), 3117-3124.

[7] T. Suzuki, Generalized distance and existence theorems in complete metric spaces, J. Math. Anal. Appl, 253(2) (2001), 440-458.

[8] T. Suzuki, On Downing-Kirk's theorem, J. Math. Anal. Appl, 286 (2003), 453-458.

[9] T. Suzuki, Several fixed point theorems concerning $\tau$-distance, Fixed Point Theory and Applications, 3 (2004), 195-209.

[10] T. Suzuki, Generalized Caristi's fixed point theorems by Bae and othrs, J. Math. Anal. Appl, 302 (2005), 502-508.

[11] T. Suzuki, Thestrong Ekeland vriational principle, J. Math. Anal. Appl, 320 (2006), 787-794.

[12] W. Takahashi, Existence theorems generalazing fixed point theorems for multivalued mappings, "Fixed Point Theory and Applications" , 252(1991), 397-406.

[13] D. Tataru, Viscosity soluation of Hamilton-Jacbi equations with unbounded nonlinear terms, J. Math. Anal. Appl, 163 (1992), 345-392.

[14] L. B. Ćirić, Generalized contractions and fixed-point theorems, Publ. Inst. Math. (Beograd)(N.S.), 12(26) (1971), 19-26.

[15] L. B. Ćirić, On a family of contractive maps and fixed-points, Publ. Inst. Math. (Beograd)(N.S.), 17(31) (1974), 45-51.

[16] L. B. Ćirić, A generalization of Banbch's contractions principle, Proc. Amer. Math. Soc, 45 (1974), 267-273.

1 Dept. of Math., Islamic Azad University,Science and Research Branch, Tehran, Iran

2 Dept. of Math., Amirkabir University of Technology, Hafez Ave., P. O. Box 15914, TEHRAN, IRAN

E-mail address: vaez@aut.ac.ir 